\title{
Evaluation of the University of Minnesota Tri-plate and 3M Petrifilm for the isolation of Staphylococcus aureus and Streptococcus species from clinically mastitic milk samples
}

\author{
J. L. McCarron, ${ }^{* 1}$ G. P. Keefe,${ }^{*} \dagger$ S. L. McKenna, ${ }^{*}$ I. R. Dohoo, ${ }^{*} \ddagger$ and D. E. Poole ${ }^{*} \dagger$ \\ *Atlantic Veterinary College Department of Health Management, \\ †Maritime Quality Milk, and \\ ¥Centre for Veterinary Epidemiological Research, University of Prince Edward Island, Charlottetown, PEI, Canada C1A 4P3
}

\begin{abstract}
The primary objective was to compare microbiological results of the University of Minnesota Tri-plate and the 3M Petrifilm Staph Express (STX) Count Plate to standard culture techniques for identification of clinical mastitis caused by Staphylococcus aureus. The secondary objective was to evaluate the Tri-plate's ability to differentiate Streptococcus spp. from other gram-positive organisms. The tests were evaluated using clinically positive mastitic milk samples $(\mathrm{n}=282)$ to determine their ability to diagnose the pathogens of interest. A Tri-plate was classified positive for Staph. aureus when at least 1 colony exhibiting $\beta$-hemolysis was present on the Factor media portion of the plate. When the plate was used in this manner and read by a trained laboratory technician, the sensitivity of the Tri-plate was $97.9 \%$ and the specificity was $81.8 \%$. When the Tri-plate was evaluated by the laboratory technician for its ability to diagnose Streptococcus spp., both sensitivity and specificity of the test were very good (92.6 and $89.5 \%$, respectively). Using the Petrifilm, samples were classified as positive for Staph. aureus if any redviolet colonies were present on the Petrifilm after an initial 24-h incubation. When used in this manner, the Petrifilm had a sensitivity of $97.4 \%$ and a specificity of $76.1 \%$. Further evaluation of the Petrifilm was done using the STX disk, which was used to confirm the presence of Staph. aureus. When using the presence of 1 pink colony on the disk, the sensitivity of the Petrifilm was $92.1 \%$ and the specificity was $93.1 \%$. Both the Triplate and the 3M STX Petrifilm successfully diagnosed Staph. aureus in clinical milk samples when used in a laboratory setting and the Tri-plate successfully differentiated Streptococcus spp. from other gram-positive organisms.
\end{abstract}

Received April 29, 2009.

Accepted June 12, 2009

${ }^{1}$ Corresponding author: jmccarron@upei.ca
Key words: mastitis, culture, Tri-plate, Petrifilm Staph Express

\section{INTRODUCTION}

Mastitis is the most costly infectious disease in the dairy industry (Erskine et al., 2003) and as a result, much work has been done developing comprehensive control programs aimed at disease prevention. Even in situations where all preventive measures are carried out, there continues to be cases of clinical mastitis. The presence of these cases requires farms to incorporate diagnostic and treatment protocols into their overall mastitis control programs. These treatment protocols should be farm-specific and recognize the predominant pathogens present (LeBlanc et al., 2006). Knowing the pathogens present on a farm and in an individual cow will allow producers to make rational therapy decisions as well as implement specific control measures that would be best suited for the pathogen present. Continued surveillance will allow producers to detect the presence of new or emerging pathogens on the farm (Ruegg, 2003).

Rational use of antibiotics and appropriately targeted treatment strategies were the major foci of mastitis control programs (LeBlanc et al., 2006). Yet, therapy of clinical mastitis remains a topic of debate as no consistent approach to the early identification and treatment of clinical mastitis cases has been developed. Ideally, aseptically acquired milk samples obtained from each clinical case of mastitis would be cultured in a laboratory before making an individual cow treatment decision, but in many cases, and for various reasons, treatment decisions are made empirically. Leslie et al. (2002) identified the need for rapid, sensitive, specific, and inexpensive aids to categorize cases of IMI. Since then, various studies evaluated cow-side tests able to successfully categorize mastitis-causing pathogens into potential treatment categories (Ruegg, 2005; Lago et al., 2006; McCarron et al., 2009). 
Two tests with the ability to rapidly categorize clinically mastitic milk samples into broad treatment categories are the University of Minnesota Tri-plate and the $3 \mathrm{M}$ Petrifilm. Because of variation in growth requirements for some bacteria, on-farm culture systems could not effectively detect all pathogens associated with mastitis (Godden et al., 2007). The University of Minnesota Tri-plate is divided into 3 types of media. Factor medium is specific for gram-positive organisms, modified thallium sulfate-crystal violet-B toxin blood (MTKT) medium is specific for Streptococcus spp., and MacConkey medium is specific for gram-negative organisms. The Factor medium of the plate is primarily used for the identification of Staph. aureus and Staphylococcus spp. Staphylococcus aureus appeared as creamy, grayish, or golden colonies with a zone of complete hemolysis around the colony (Laboratory for Udder Health, 2000). Testing for the presence and type of hemolysis on blood agar-based plates represented a first simple and rapid method for the detection of Staph. aureus in milk samples (Lam et al., 1995). However, previous research showed that approximately 20 to $25 \%$ of the Staph. aureus isolates from bovine mastitis do not show detectable $\beta$-hemolysis activity in primary cultures, making this a specific but not very sensitive criterion for the identification of Staph. aureus (Boerlin et al., 2003).

The 3M Petrifilm Staph Express Count Plate (STX) is designed to be selective and differential for Staphylococcus spp. The Petrifilm produces results within 24 $\mathrm{h} \pm 2 \mathrm{~h}$ of incubation. A further $3 \mathrm{~h}$ of incubation is required when the confirmatory STX Disk is applied (3M Microbiology, 2005). Rapid results may be preferred in situations where timely decisions are required, such as in on-farm culture programs (Silva et al., 2005). Many studies noted that sensitive and rapid cow-side diagnostic kits for the identification of Staph. aureus in milk could play an important role in the control of contagious mastitis (Hogan et al., 1986; Watts and Owens, 1988; Boerlin et al., 2003).

The primary objective was to compare microbiological results of the University of Minnesota Tri-plate and the 3M Petrifilm STX to standard culture techniques for identification of Staph. aureus in clinically mastitic milk samples. The secondary objective was to evaluate the Tri-plate's ability to differentiate Streptococcus spp. from other gram-positive organisms.

\section{MATERIALS AND METHODS}

Details of sample collection and processing, including laboratory procedures for gold standard testing, were presented in a previous paper (McCarron et al., 2009). A brief summary of the procedures is presented below.

\section{Samples}

Samples of clinically mastitic milk $(\mathrm{n}=282)$ were collected from 21 dairy farms in Prince Edward Island. Samples with complete records $(\mathrm{n}=271)$ were used for Tri-plate evaluation and a subset $(\mathrm{n}=213)$ was used for evaluation of the Petrifilm STX plate. Producers were trained to recognize abnormal (mastitic) milk and aseptically collect all milk samples. Samples were never frozen, but were kept refrigerated and transported to the Atlantic Veterinary College for culture within 24 to $36 \mathrm{~h}$ of collection.

\section{Gold Standard}

Gold standard bacteriological cultures were performed according to the Laboratory Handbook on Bovine Mastitis (National Mastitis Council, 1987). Samples were classified as having significant growth if the growth was considered of "probable significance" or "highly significant" based on National Mastitis Council Guidelines for significance (National Mastitis Council, 1987). Disposable plastic loops were used to streak 10 $\mu \mathrm{L}$ of each sample onto blood agar and MacConkey plates. Plates were incubated at $35^{\circ} \mathrm{C}$ for $24 \mathrm{~h}$. The standard laboratory plates were read by a milk laboratory technician, and colonies were identified based on growth characteristics, morphology, pattern of hemolysis, catalase reaction, and Gram-staining properties. The tube coagulase test was used to differentiate Staph. aureus from other CNS species. Samples that grew a yeast or mold were classified as no bacterial growth. Samples that had 2 colony types were considered mixed growth and samples with 3 or more were considered contaminated. If samples exhibited mixed growth or contamination on gold standard culture, they were not used in the evaluation of the Tri-plate and Petrifilm.

\section{Minnesota Easy Culture System II Tri-plate}

The Minnesota Easy Culture System II Tri-plate, developed by the University of Minnesota's Laboratory for Udder Health (St. Paul, MN), is a culture plate that is divided in 3 sections. One section contains a proprietary Factor medium that is selective for gram-positive bacteria, 1 section contains MacConkey medium for the identification of gram-negative bacteria, and 1 section contains MTKT medium that is selective for Streptococcus spp. (Laboratory for Udder Health, 2000). The media were inoculated according to the manufacturer's recommendations. Sterile cotton-tipped swabs were saturated in milk and used to inoculate each section of the plate, re-dipping the swab in the sample before inoculating each section. Plates were incubated in a $35^{\circ} \mathrm{C}$ incubator for $24 \mathrm{~h}$ before being read. 
The criterion used to classify a sample as positive for Staph. aureus was growth on the Factor media that exhibited $\beta$-hemolysis around at least 1 colony. One colony of Staph. aureus is considered significant by the National Mastitis Council, so plates were assigned to the Staph. aureus category if a single colony exhibiting $\beta$-hemolysis was present. If there was growth on the Factor medium that did not exhibit $\beta$-hemolysis, growth on either of the other media, or no growth on any media, the sample was assigned to the Staph. aureus-negative category. Each Tri-plate was read by a trained milk microbiology technician as well as 4 masked readers with limited microbiology experience to determine their ability to detect Staph. aureus by identifying $\beta$-hemolysis on Factor media.

Tri-plates were evaluated for their ability to diagnose Streptococcus spp. Plates were considered positive for Streptococcus spp. if there was colony growth (at least 1 colony) on the MTKT media. Again, each Tri-plate was read by a trained milk microbiology technician as well as 4 masked readers with limited microbiology experience.

\section{M Petrifilm Staph Express}

The second media system used was the $3 \mathrm{M}$ Petrifilm STX. The Petrifilm STX is a sample-ready culture medium system, which contains a cold-water-soluble gelling agent. The chromogenic, modified Baird-Parker medium in the plate is selective and differential for Staph. aureus. Red-violet colonies on the plate are Staph. aureus (3M Microbiology, 2005). In cases where the color of the colonies was not easily identified or when colonies other than red-violet were present on the plate, the 3M Petrifilm STX disk may be used to identify Staph. aureus. The STX disk contained a dye and DNA. Staphylococcus aureus produces DNase that reacts with the dye to form pink zones. Milk samples were diluted 1:10 with sterile water, a 1-mL aliquot was plated on each STX Petrifilm, and plates were incubated at $35^{\circ} \mathrm{C}$ for $24 \mathrm{~h}$. Colony growth, number of colonies present, and color of colonies were recorded by the laboratory technician. Initial test characteristics (sensitivity, specificity, and predictive values) were calculated based solely on the presence of red-violet colonies as per the manufacturer's interpretation criteria. Subsequently, all Petrifilms that were positive for growth (at least 1 colony) had the STX disk inserted into the plate and were re-incubated at $37^{\circ} \mathrm{C}$ for $3 \mathrm{~h}(\mathrm{n}$ $=123$ ). Plates that exhibited at least 1 colony with a pink zone were classified positive for Staph. aureus. Pink colonies were then picked and re-grown using standard methods to confirm their identity.
All Petrifilms were read by the 4 masked readers and the laboratory technician; however, after the STX disks were applied, plates were read only by the laboratory technician; therefore, no inter-reader comparisons were made.

\section{Statistical Analysis}

All results were analyzed using Intercooled Stata 9 (StataCorp, 2005). For assessment of the Tri-plate system's ability to identify Staph. aureus and Streptococcus spp., sensitivity (Se), specificity (Sp), positive predictive value (PPV), and negative predictive value (NPV) were calculated by comparing the Triplate classifications to the gold standard results. The Petrifilm STX was evaluated in the same manner to determine its ability to diagnose Staph. aureus.

\section{RESULTS}

\section{Samples}

A total number of 282 fresh milk samples were received from producers. Three samples had incomplete records and 18 samples were removed from the analysis because they exhibited mixed or contaminated growth on the gold standard results, leaving 261 samples for Tri-plate analysis. Gold standard culture results for each sample used in the analysis are in Table 1. The prevalence of Staph. aureus used to evaluate the Triplates was $18.0 \%$ and the prevalence of all Strep. spp. combined was $30.7 \%$.

In total, 213 samples had complete records on the Petrifilm STX. After 6 wk of sample collection, the laboratory technician noted that $1 \mathrm{~mL}$ of undiluted milk plated on the Petrifilms resulted in some plates that were difficult to interpret (milk clots and cases of heavy bacterial growth resulted in difficulty identifying individual colonies). At that point, a second series of Petrifilms with a 1 in 10 dilution (equivalent to $100 \mu \mathrm{L}$ of milk and $900 \mu \mathrm{L}$ of sterile diluent on the film) was included. There were 64 samples received at the beginning of the collection period that were not diluted, leaving 213 samples with complete records. Sixteen were not included in the final analysis, because they were contaminated on the gold standard culture. Previous research evaluating Petrifilm Aerobic Count and Coliform Count performance using diluted versus undiluted milk showed no difference in Se and NPV, but statistically higher Sp and PPV when diluted milk was used (McCarron et al., 2009). Wallace et al. (2008) found test characteristics of the STX were the highest for diluted (1 in 10) fresh samples from clinically mastitic 
Table 1. Gold standard microbiology results of samples used to evaluate the University of Minnesota Tri-plate and the 3M Petrifilm Staph Express

\begin{tabular}{|c|c|c|c|c|}
\hline Gold standard result & $\begin{array}{l}\text { Samples used for } \\
\text { Tri-plate evaluation }\end{array}$ & Percentage & $\begin{array}{l}\text { Samples used for } \\
\text { Petrifilm evaluation }\end{array}$ & Percentage \\
\hline No bacterial growth & 85 & 32.6 & 61 & 30.1 \\
\hline Staphylococcus aureus & 47 & 18.0 & 38 & 19.3 \\
\hline Streptococcus uberis & 43 & 16.5 & 36 & 18.3 \\
\hline Escherichia coli & 27 & 10.3 & 18 & 9.1 \\
\hline Streptococcus spp. & 18 & 6.9 & 11 & 5.6 \\
\hline Streptococcus dysgalactiae & 18 & 6.9 & 13 & 6.6 \\
\hline CNS spp. & 6 & 2.3 & 5 & 2.5 \\
\hline Staphylococcus spp. & 4 & 1.5 & 4 & 2.0 \\
\hline Pasteurella multocida & 3 & 1.2 & 3 & 1.5 \\
\hline Staphylococcus hyicus & 3 & 1.2 & 3 & 1.5 \\
\hline Klebsiella spp. & 2 & 0.8 & 2 & 1.0 \\
\hline Streptococcus bovis & 1 & 0.4 & 0 & 0 \\
\hline Bacillus spp. & 1 & 0.4 & 0 & 0 \\
\hline Corynebacterium spp. & 1 & 0.4 & 1 & 0.5 \\
\hline Serratia spp. & 1 & 0.4 & 1 & 0.5 \\
\hline Enterobacter spp. & 1 & 0.4 & 1 & 0.5 \\
\hline Total & 261 & 100 & 197 & 100 \\
\hline
\end{tabular}

cows. For these reasons, only diluted milk samples were included in the analysis. Table 1 illustrates the gold standard culture results for each of the samples used to determine test characteristics of the STX Petrifilm. The prevalence of Staph. aureus in the samples used to evaluate the Petrifilm was $19.3 \%$.

\section{Minnesota Easy Culture System II Tri-plate}

Table 2 illustrates the test characteristics of the Triplate when read by the laboratory technician. The Triplate was highly sensitive because only 1 sample that was positive for Staph. aureus on the gold standard was not diagnosed using the Tri-plate. The reduced Sp was the result of 39 samples being classified as Staph. aureus by the Tri-plate that were not diagnosed as Staph. aureus by the gold standard methods. Most (12) of these samples had no bacterial growth on the gold standard, the remaining samples included 9 Streptococcus uberis, 7 Streptococcus spp., 5 Streptococcus dysgalactiae, 5 Escherichia coli, and 1 Staphylococcus spp.

Table 3 shows the test characteristics of the Triplates when read by the 4 masked readers who used a threshold of 1 colony that exhibited $\beta$-hemolysis on Factor media to consider a plate positive for Staph. aureus. The Se of the Tri-plate was lower when read by the readers (43.2 to $59.1 \%$ ) with limited microbiology training than when read by the technician. The level of agreement among the readers was moderate, with the calculated kappa value (actual agreement beyond chance) being 0.51 . The Sp of the test was higher with the inexperienced readers, ranging from 93.8 to $95.9 \%$. All test characteristics calculated were similar between the readers with no significant differences observed.

Table 4 shows the test characteristics of the Triplate when evaluated by the laboratory technician for its ability to diagnose a Streptococcus sp. Sensitivity and $\mathrm{Sp}$ of the test were 92.6 and $89.5 \%$, respectively. Six samples that were diagnosed as Streptococcus spp. using the gold standard did not grow on the MTKT media. Nineteen samples that exhibited growth on the MTKT medium were not diagnosed as Streptococcus spp. using the gold standard. On the gold standard, 9 of the 19 were diagnosed no growth, 5 Staph. aureus, 3 E. coli, 1 Corynebacterium spp., and 1 Staphylococcus spp. The same masked readers evaluated the Tri-plate for the presence of Streptococcus spp. The sensitivities of the Tri-plate, when read by these readers, ranged from 77.8 to $88.9 \%$ and specificities from 83.7 to $92.7 \%$ with a combined kappa value of 0.81 .

\section{M Petrifilm Staph Express}

Samples were classified as positive for Staph. aureus if any red-violet colonies were present on the Petrifilm after the initial $24 \mathrm{~h}$ incubation. Test characteristics and predictive values are presented in Table 5. When used in this manner the Petrifilm was highly sensitive $(97.4 \%)$ but not very specific (76.1\%). Only 1 sample that was diagnosed Staph. aureus using the gold standard did not show growth of red-violet colonies. There were 38 samples that grew red-violet colonies, but were

Table 2. Test characteristics of the University of Minnesota Triplate when read by an experienced technician based on its ability to correctly classify samples as Staphylococcus aureus

\begin{tabular}{lc}
\hline Test characteristic & Value $(95 \%$ confidence interval) \\
\hline Sensitivity, \% & $97.9(96.1-99.6)$ \\
Specificity, \% & $81.8(77.1-86.5)$ \\
Positive predictive value, \% & $54.1(48.1-60.2)$ \\
Negative predictive value, \% & $99.4(98.5-100)$ \\
\hline
\end{tabular}


Table 3. Test characteristics of the University of Minnesota Tri-plate based on its ability to correctly classify samples as Staphylococcus aureus when read by masked readers with limited microbiology training

Value (95\% confidence interval)

\begin{tabular}{lcccc}
\cline { 2 - 5 } Test characteristic & Reader A & Reader B & Reader C & Reader D \\
\hline Sensitivity, \% & $51.1(45.0-57.3)$ & $43.2(37.1-49.3)$ & $54.6(48.4-60.7)$ & $59.1(52.9-65.3)$ \\
Specificity, \% & $93.8(90.9-96.8)$ & $95.2(92.6-97.9)$ & $94.8(92.0-97.5)$ & $95.9(93.4-98.4)$ \\
Positive predictive value, \% & $63.9(58.0-69.8)$ & $65.5(59.7-71.4)$ & $68.6(62.9-74.3)$ & $76.5(71.1-81.8)$ \\
Negative predictive value, \% & $90.0(86.3-93.6)$ & $88.8(85.0-92.3)$ & $90.9(87.3-94.4)$ & $91.3(87.7-94.8)$ \\
\hline
\end{tabular}

not classified Staph. aureus using the gold standard culture. Eight of these samples showed no growth on the gold standard culture, 16 were Streptococcus spp., 7 were Staphylococcus spp., 6 were gram-negative coliforms, and 1 was Corynebacterium bovis.

Table 5 illustrates the test characteristics of the Petrifilm STX after the application of the STX disk and reincubation for $3 \mathrm{~h}$. All plates were read only by the laboratory technician, and the presence of 1 pink colony was used classify a sample as positive for Staph. aureus. The Petrifilm STX with disk applied was highly sensitive (92.1\%) because only 3 of the 38 samples that were positive for Staph. aureus on the gold standard were not detected using the STX Petrifilm. Two of the 3 samples did not show any visible growth on the Petrifilm and the remaining sample did show colony growth, but was not confirmed pink using the disk. The Sp of the test was significantly increased when the STX disk was used (from 76.1 to $93.1 \%$ ). This was the result of only 11 samples being classified as Staph. aureus by the Petrifilm STX with disk applied that were not diagnosed as Staph. aureus using the gold standard. Each of the 11 samples had the pink colonies picked from the plate and recultured using standard methods. Five were confirmed Staph. aureus, 4 were Staphylococcus spp., 1 Staph. hyicus, and 1 yielded no growth. Had the 5 samples that were diagnosed as Staph. aureus on subsequent standard culture methods been diagnosed by the original gold standard culture the Se of the Petrifilm would be increased to $93.0 \%$ and the PPV would increase to $87.0 \%$.

When based on results of the STX Petrifilm using the presence of red-violet colonies to diagnose Staph. aureus, the apparent prevalence was $38.1 \%$ and when the diagnosis was based on confirmation by the STX disk, the apparent prevalence of Staph. aureus was $23.4 \%$. The application of the disk did not result in any new samples being identified as Staph. aureus positive.

\section{DISCUSSION}

A complete mastitis control program should include the use of microbiologic analysis of individual milk samples to determine which mastitis pathogens are present on the farm (Ruegg, 2003). Having on-farm access to accurate, rapid microbiological tests that are capable of diagnosing some of the most prevalent mastitis pathogens may facilitate the incorporation of this valuable data into mastitis control programs.

When implementing preventative strategies for mastitis, information about the prevalence of specific pathogens is useful (Makovec and Ruegg, 2003). The National Mastitis Council Recommended Mastitis Control Program suggests milking cows with confirmed contagious IMI last and marketing or permanently segregating cows that are persistently infected with Staph. aureus or other nonresponsive microbial agents. Confirming the presence of Staph. aureus quickly and accurately may allow better execution of segregation strategies. For producers making decisions on treatment protocols (routine, extended therapy, withholding treatment, and culling), identification of Staph. aureus is of value (Sol et al., 2000).

\section{Samples}

The 2 mastitis-causing pathogens that are the focus here are Staph. aureus and Streptococcus spp. Both of these pathogens are considered significant mastitiscausing organisms by the National Mastitis Council (National Mastitis Council, 1987) and are 2 of the most prevalent pathogens encountered. For epidemiologic monitoring and investigation of risk factors for prevention of cases, identification of the type of gram-positive organism (Streptococcus spp. or Staph. aureus) is important. Streptococcus spp. were isolated from $12.2 \%$

Table 4. Test characteristics of the University of Minnesota Triplate when read by an experienced technician based on its ability to correctly classify samples as Streptococcus spp.

\begin{tabular}{lc}
\hline Test characteristic & Value $(95 \%$ confidence interval) \\
\hline Sensitivity, \% & $92.6(89.4-95.8)$ \\
Specificity, \% & $89.5(85.8-93.2)$ \\
Positive predictive value, \% & $79.8(74.9-84.7)$ \\
Negative predictive value, \% & $96.4(94.2-98.7)$ \\
\hline
\end{tabular}


Table 5. Test characteristics of the 3M Staph Express Petrifilm when read by an experienced technician using the presence of red-violet colonies on initial culture to classify samples as Staphylococcus aureus versus using the confirmatory disk

\begin{tabular}{lcc}
\hline & \multicolumn{2}{c}{ Value $(95 \%$ confidence interval) } \\
\cline { 2 - 3 } Test characteristic & Red-violet colonies & Staph Express disk \\
\hline Sensitivity, \% & $97.4(95.1-99.6)$ & $92.1(88.3-95.9)$ \\
Specificity, \% & $76.1(70.2-82.1)$ & $93.1(89.5-96.6)$ \\
Positive predictive value, \% & $49.3(42.4-56.3)$ & $76.1(70.1-82.0)$ \\
Negative predictive value, \% & $99.2(97.9-100)$ & $98.0(96.1-100)$ \\
\hline
\end{tabular}

of milk samples submitted to the Wisconsin Veterinary Diagnostic Laboratory and Staph. aureus were isolated from $9.7 \%$ of samples (Makovec and Ruegg, 2003). More recently, a Canadian prevalence study of clinical mastitis found $12.5 \%$ of samples submitted were positive for Streptococcus Spp. and $10.3 \%$ positive for Staph. aureus (Olde Riekerink et al., 2008). Here, Streptococcus spp. were the most prevalent species cultured. Of the 261 samples used to evaluate the Tri-plate, $30.7 \%$ grew Streptococcus spp. on the gold standard culture. Staphylococcus aureus was the second most prevalent organism cultured on the gold standard, with a prevalence of $18.0 \%$. In the 197 samples used to evaluate the Petrifilm, $30.5 \%$ were diagnosed as Streptococcus spp. on the gold standard and $19.3 \%$ were diagnosed as Staph. aureus.

\section{Minnesota Easy Culture System II Tri-plate}

The Tri-plate culture system was designed to identify some of the most common pathogens infecting the bovine udder (Bey and Farnsworth, 2000). Godden et al. (2007) evaluated the system in laboratory and field studies for its ability to differentiate growth from no growth, gram-positive from gram-negative and growth of Staphylococcus versus Streptococcus spp. They found the system accurate and attained a high level of agreement (kappa values between 0.80 and 0.93 ) when used for those 3 purposes. However, when users tried to differentiate pathogen groups further or to identify specific pathogens (Staph. aureus vs. CNS spp.) much lower agreement was observed. When the University of Minnesota Bi-plate (which uses Factor and MacConkey media) was evaluated for its ability to differentiate growth from no growth and gram-positive from gram-negative organisms, the test was highly sensitive (97.9\%; McCarron et al., 2009). Similarly, agreement among readers was high (kappa $=0.76$ ) when asked to differentiate the above categories. In the current research, when readers were asked to indentify Staph. aureus on the Tri-plate, in concurrence with Godden et al. (1997), the agreement among readers was low $($ kappa $=0.51)$.
$\beta$-Hemolysis represents an important criterion for rapid presumptive identification of Staph. aureus in primary cultures (Boerlin et al., 2003). Of the 3 coagulase-positive Staphylococcus spp. regularly encountered in clinical milk samples, Staph. aureus was the only one with hemolytic activity (Lam et al., 1995). Yet, 20 to $25 \%$ of the Staph. aureus isolates from bovine mastitis did not present detectable $\beta$-hemolysis in primary cultures. The proportion of $\beta$-hemolytic Staph. aureus found in bovine mastitis varies from region to region (Larsen et al., 2002). Therefore, the performance of a test that uses hemolysis as part of the diagnostic criteria may vary with regards to the population under investigation (Boerlin et al., 2003). In this study, the Tri-plate was highly sensitive $(97.9 \%)$ with moderate Sp (81.8\%) when used by a laboratory technician to diagnose the presence of Staph. aureus. But, when read by masked readers, the sensitivities were much lower (ranging from 43.2 to $59.1 \%$ ). In contrast, the Sp of the test was higher for the inexperienced readers, ranging from 93.8 to $95.9 \%$, possibly because these readers were more reluctant to call a colony positive, resulting in fewer false positives. Similar work by Lam et al. (1995) used the presence or absence of $\beta$-hemolysin production as a method to diagnose Staph. aureus. Plates were read by an experienced observer and 45 out of 54 samples were correctly identified, resulting in a Se of $83 \%$ and a Sp of $98 \%$. Predictive values of the test were calculated at various levels of prevalence. At a prevalence of $21 \%$ (the median prevalence among the herds in their study) the PPV was $91 \%$ and the NPV was $96 \%$.

When done by the inexperienced readers, observation of hemolysis resulted in a highly specific test with very high negative predictive values. Farm-based users of the Tri-plate in this manner could be confident in detecting animals that are negative for Staph. aureus and, depending on the prevalence in the herd, could be very confident in a negative test result. Unfortunately, if this test were used to detect positive cows for segregation from the herd, a user without extensive microbiological experience would miss several positive animals. The very high Se obtained by the laboratory technician indicates that with thorough training, the 
Tri- plate can be used to identify animals truly infected with Staph. aureus in a farm situation.

Tri-plates were evaluated for their ability to diagnose Streptococcus spp. based on the presence of colony growth on the MTKT media. When read by the technician, both the Se and Sp of the test were high (92.6 and $89.5 \%$, respectively). The lower $\mathrm{Sp}$ was the result of 19 samples showing growth on the MTKT media that were not diagnosed as Streptococcus spp. on the gold standard. Almost half (9) of the samples showed no growth on the gold standard. This may be due to a larger inoculum of milk used on the Tri-plate (one saturated cotton swab for one-third of the plate versus the $10-\mu \mathrm{L}$ loop used for the gold standard). Five samples that were diagnosed as Staph. aureus on the gold standard exhibited colony growth on MTKT and were therefore considered Streptococcus spp.-positive. The presence of 1 colony of Staph. aureus in the gold standard, regardless of the presence of other colony types, resulted in a diagnosis of Staph. aureus. The MTKT-positive samples may have been mixed Streptococcus spp. and Staph. aureus infections.

\section{M Petrifilm Staph Express}

The Petrifilm STX plate was designed to provide rapid results for the diagnosis of Staph. aureus after $24 \pm 2 \mathrm{~h}$ of incubation when red-violet colonies are present. The Petrifilm STX Disk should be applied to the plate and reincubated for $3 \mathrm{~h}$ whenever colonies other than red-violet are present on the plate; for example, black or blue-green colonies (3M Microbiology, 2005). The manufacturer's interpretive criteria suggest that the appearance of red-violet colonies on the initial incubation is presumptive evidence for the diagnosis of Staph. aureus. Results from Silva et al. (2005) did not support this recommendation and found it necessary to use the Staph Express Disk for confirmation even when red-violet colonies were the only ones present. They found that the apparent prevalence of Staph. aureus in milk samples processed using Petrifilm was significantly greater than the prevalence in milk samples processed using standard microbiological techniques. The results of the current study show that the STX Petrifilm had a higher Se when using only the presence of red-violet colonies than when the presence of pink colonies on the Staph Express Disk was used, 97.4 versus 92.1\%. In the population of samples that were used in this study, only 38 were diagnosed as Staph. aureus on the gold standard. The difference in sensitivities was due to the difference in classification of only 2 samples; therefore, this difference may be of little biological significance. The high sensitivities obtained in both methods of evaluation indicate that using the STX Petrifilm resulted in very few false-negative classifications. This is an important attribute for a test to have if it were being used as part of a mastitis control program because the occurrence of false-negative results could result in the maintenance of infected animals within a herd (Silva et al., 2005). The Sp of the STX Petrifilm was greatly improved by the use of the STX disk (from 76.1 to 93.1\%). Results from Silva et al. (2005) showed that the STX Petrifilm was highly specific $(98.5 \%)$ when the disk was used. In the current study, the Sp of using the presence of red-violet colonies was decreased because 38 samples that were diagnosed as Staph. aureus using the Petrifilm were not Staph. aureus on the gold standard. These results show that a variety of organisms other than Staph. aureus will grow red-violet on the STX Petrifilm; therefore, caution should be used when using this test without the confirmatory disk, especially if segregation or culling decisions are made based on the results. The application of the STX disk did not improve the Se of the test as the manufacturer suggests, rather the $\mathrm{Sp}$ of the test was greatly improved.

In the case of $\beta$-hemolysis on the Tri-plate, where previous experience of the reader would potentially affect the outcome of the test, readers with extensive experience and inexperienced readers were used. Our findings, along with results of other studies that have evaluated potential on-farm tests for mastitis, support the need for thorough training of individuals reading the tests. Previously published work also recommends that periodic assessment of accuracy of on-farm methods by submission of duplicate samples to a microbiology laboratory be carried out (Silva et al., 2005).

When the 2 tests were read by the laboratory technician, there was no significant difference in the detection of Staph. aureus (Se). The specificities of the 2 tests were similar when the presence of red-violet colonies only was used to assess the Petrifilm, but with the application of the STX disk, the Petrifilm was a more specific test than the Tri-plate.

\section{CONCLUSIONS}

Both the University of Minnesota Tri-plate and the 3M STX Petrifilm were able to successfully detect Staph. aureus in clinical milk samples when used in a laboratory setting. The presence of $\beta$-hemolysis on the Tri-plate was a highly sensitive method to diagnose Staph. aureus when read by a trained laboratory technician. However, when read by individuals with limited microbiology experience, the Se of the test was much lower. Specificity of the Tri-plate system was higher when read by the inexperienced readers than by the 
laboratory technician. The Tri-plate was able to successfully differentiate Streptococcus spp. from other gram-positive organisms.

The 3M Petrifilm was very sensitive when used to diagnose the presence of Staph. aureus. When using the presence of red-violet colonies only to diagnose a sample positive with Staph. aureus, the Se and Sp of the Petrifilm were similar to those of the Tri-plate. The Sp of the Petrifilm was increased significantly by using the STX confirmatory disk.

To determine their suitability as on-farm tests for specific mastitis-causing pathogens, each of these tests should be evaluated in field situations. Training of individuals performing and reading the tests will be key to their success on the farm. For quality control purposes, samples taken on the farm should be saved and submitted to a diagnostic laboratory periodically to assess the ongoing accuracy of any on-farm culture system.

\section{ACKNOWLEDGMENTS}

The authors thank T. Andrews, L. Dalziel, K. MacIntosh, M. Trenholm, C. Mitchell, and S. Richard of the Department of Health Management (University of Prince Edward Island, Charlottetown, PEI, Canada) for their technical help with this project.

This research was financed by Natural Sciences and Engineering Research Council of Canada (Ottawa, Ontario, Canada); Alberta Milk (Edmonton, AB, Canada); Dairy Farmers of New Brunswick (Sussex, NB, Canada), Nova Scotia (Truro, NS, Canada), Ontario (Mississauga, ON, Canada), and Prince Edward Island (Charlottetown, PEI, Canada); Novalait Inc. (Sainte-Foy, PQ, Canada); Dairy Farmers of Canada (Ottawa, Ontario, Canada); Canadian Dairy Network (Guelph, Ontario, Canada); Agriculture and Agri-Food Canada (Ottawa, Ontario); Public Health Agency of Canada (Ottawa, Ontario); Technology PEI Inc. (Charlottetown, PEI, Canada); Université de Montréal (St. Hyacinthe, PQ, Canada); and University of Prince Edward Island (Charlottetown, PEI, Canada) through the Canadian Bovine Mastitis Research Network (St. Hyacinthe, PQ, Canada).

\section{REFERENCES}

3M Microbiology. 2005. 3M Petrifilm Interpretation Guide. 3M Microbiology, St. Paul, MN.

Bey, R., and R. Farnsworth. 2000. Cow-side microbiology. Page 97 in Proc. Annu. Mtg. Minnesota Dairy Health Management Conf., St. Paul, MN.

Boerlin, P., P. Kuhnert, D. Hussy, and M. Schaellibaum. 2003. Methods for identification of Staphylococcus aureus isolates in cases of bovine mastitis. J. Clin. Microbiol. 41:767-771.
Erskine, R. J., S. Wagner, and F. J. DeGraves. 2003. Mastitis therapy and pharmacology. Vet. Clin. North Am. Food Anim. Pract. 19:109-138.

Godden, S., A. Lago, R. Bey, K. Leslie, P. Ruegg, and R. Dingwell. 2007. Use of on-farm culture systems in mastitis control programs. Page 1-9 in Natl. Mastitis Counc. Reg. Mtg. Proc., Visalia, CA.

Hogan, J. S., A. Cornetta, and J. W. Pankey. 1986. Comparison of four test procedures to identify Staphylococcus aureus isolated from bovine intramammary infections. Am. J. Vet. Res. 47:20172019.

Laboratory for Udder Health. 2000. Minnesota Easy Culture System II Handbook. University of Minnesota Veterinary Diagnostic Laboratory, St. Paul, MN

Lago, A., S. Godden, R. Bey, K. Leslie, R. Dingwell, and P. Ruegg. 2006. Validation of the Minnesota Easy Culture System II: Results from on-farm bi-plate culture versus standard laboratory culture. Page 250-251 in Am. Assoc. Bov. Prac. Ann. Mtg. Proc., St. Paul, MN.

Lam, T. J. G. M., A. Pengov, Y. H. Schukken, J. A. H. Smit, and A. Brand. 1995. The differentiation of Staphylococcus aureus from other micrococcaceae isolated from bovine mammary glands. J. Appl. Bacteriol. 79:69-72.

Larsen, H. D., F. M. Aarestrup, and N. E. Jensen. 2002. Geographical variation in the prevalence of genes encoding superantigenic exotoxins and $\beta$-hemolysin among Staphylococcus aureus isolated from bovine mastitis in Europe and USA. Vet. Microbiol. 85:6167.

LeBlanc, S. J., K. D. Lissemore, D. F. Kelton, T. F. Duffield, and K. E. Leslie. 2006. Major advances in disease prevention in dairy cattle. J. Dairy Sci. 89:1267-1279.

Leslie, K. E., J. T. Jansen, and G. H. Lim. 2002. Opportunities and implications for improved on-farm cowside diagnostics. Page 147160 in Proc. DeLaval Hygiene Symp., Guelph, ON, Canada.

Makovec, J. A., and P. L. Ruegg. 2003. Results of milk samples submitted for microbiological examination in Wisconsin from 1994 to 2001. J. Dairy Sci. 86:3466-3472.

McCarron, J. L., G. P. Keefe, S. L. McKenna, I. R. Dohoo, and D. E. Poole. 2009. Laboratory evaluation of 3M Petrifilms and University of Minnesota Bi-plates as potential on-farm tests for clinical mastitis. J. Dairy Sci. 92:2297-2305.

National Mastitis Council. 1987. Laboratory and Field Handbook on Bovine Mastitis. 1st ed. W. D. Hoard and Sons Co., Fort Atkinson, WI.

Olde Riekerink, R. G., H. W. Barkema, D. F. Kelton, and D. T. Scholl. 2008. Incidence rate of clinical mastitis on Canadian dairy farms. J. Dairy Sci. 91:1366-1377.

Ruegg, P. 2005. On-farm diagnosis of mastitis infections and organisms. Page 24-30 in Natl. Mast. Counc. Reg. Mtg. Proc., Burlington, VT.

Ruegg, P. L. 2003. Investigation of mastitis problems on farms. Vet. Clin. North Am. Food Anim. Pract. 19:47-73.

Silva, B. O., D. Z. Caraviello, A. C. Rodrigues, and P. L. Ruegg. 2005. Evaluation of Petrifilm for the isolation of Staphylococcus aureus from milk samples. J. Dairy Sci. 88:3000-3008.

Sol, J., O. C. Sampimon, H. W. Barkema, and Y. H. Schukken. 2000. Factors associated with cure after therapy of clinical mastitis caused by Staphylococcus aureus. J. Dairy Sci. 83:278-284.

StataCorp. 2005. Intercooled Stata 9.0. StataCorp, College Station, TX.

Wallace, J., J. Roy, E. Bouchard, L. DesCoteaux, S. Messier and D. DuTremblay. 2008. Comparison of 3M Petrifilm Staph Express Count plates, 3M Petrifilm Rapid Coliform Count plates and 3M Aerobic Count plates with standard bacteriology of bovine milk. Page 162-163 in Natl. Mast. Counc. Annu. Mtg., New Orleans, LA.

Watts, J. L., and W. E. Owens. 1988. Evaluation of the rapid mastitis test for identification of Staphylococcus aureus and Streptococcus agalactiae isolated from bovine mammary glands. J. Clin. Microbiol. 26:672-674. 